\title{
Fixed Points in Topological *-Algebras of Unbounded Operators
}

\author{
By
}

Fabio BAGARELLO*

\begin{abstract}
We discuss some results concerning fixed point equations in the setting of topological *-algebras of unbounded operators. In particular, an existence result is obtained for what we have called weak $\tau$ strict contractions, and some continuity properties of these maps are discussed. We also discuss possible applications of our procedure to quantum mechanical systems.
\end{abstract}

\section{$\S 1$. Introduction and Mathematical Framework}

Fixed point theorems have often proved to be powerful tools for abstract analysis as well as for concrete applications, see [1], [2], [3] for general overviews. In particular, contraction mappings have been successfully used in quantum mechanics for the description of systems with infinite degrees of freedom, $Q M_{\infty}$, see [2], Section 5.6.c, and [4]. In this last reference, for instance, the existence of an (unique) fixed point has been used in the analysis of the thermodynamical limit of (a class of) mean field spin models.

On a different side, it is well known to all the people working on the algebraic approach to $Q M_{\infty}$, [5], that $C^{*}$ or Von Neumann algebras are not reach enough to be useful in the description of many physically relevant systems. For instance, difficulties already arise in ordinary quantum mechanics, since the commutation rule $[x, p]=i$ implies that the operators $x$ and $p$ cannot be both bounded as operators on $\mathcal{L}^{2}(\mathbb{R})$. These physical difficulties have originated

Communicated by T. Kawai, July 14, 2000. Revised January 16, 2001.

2000 Mathematics Subject Classification(s): 46N50, 47H10.

* Dipartimento di Matematica ed Applicazioni, Fac. Ingegneria, Università di Palermo, I-90128 Palermo, Italy.

e-mail: bagarell@unipa.it 
a wide literature about unbounded operators and possible extensions of $C^{*}$ algebras: quasi *-algebras, [6], partial *-algebras, [7], $C Q^{*}$-algebras, [8], etc. As for physical applications of these structures to $Q M_{\infty}$, some are given in [9], [10], [11], [12].

In view of these considerations, it is natural to extend the notion of contraction mappings to quasi *-algebras, and then consider the consequences of this extension.

Before giving our definition of contraction mappings and in order to keep the paper self-contained, we briefly review some relevant definitions concerning quasi $*$-algebras.

Let $\mathcal{H}$ be a Hilbert space and $N$ an unbounded, self adjoint operator defined on a dense domain $D(N) \subset \mathcal{H}$. Let $D\left(N^{k}\right)$ be the domain of the operator $N^{k}$, $k \in \mathbb{N}$, and $\mathcal{D}$ the domain of all the powers of $N$ :

$$
\mathcal{D} \equiv D^{\infty}(N)=\cap_{k \geq 0} D\left(N^{k}\right) .
$$

To be concrete we take here $N$ as the number operator for bosons, $N=a^{\dagger} a, a$ and $a^{\dagger}$ being the annihilation and creation operators satisfying the commutation relation $\left[a, a^{\dagger}\right]=\mathcal{I}$.

$\mathcal{D}$ is dense in $\mathcal{H}$. Following Lassner, [6], we define the ${ }^{*}$-algebra $\mathcal{L}^{+}(\mathcal{D})$ of all the closable operators defined on $\mathcal{D}$ which, together with their adjoints, map $\mathcal{D}$ into itself. It is clear that all the powers of $a$ and $a^{\dagger}$ belong to this set.

We define on $\mathcal{D}$ a topology $t$ by means of the following seminorms:

$$
\phi \in \mathcal{D} \rightarrow\|\phi\|_{n} \equiv\left\|N^{n} \phi\right\|,
$$

where $n$ is a natural integer and \|\| is the norm of $\mathcal{H}$, [6]. The topology $\tau$ in $\mathcal{L}^{+}(\mathcal{D})$ is given as follows: we start introducing the set $\mathcal{C}$ of all the positive, bounded and continuous functions $f(x)$ on $\mathbb{R}_{+}$, such that

$$
\sup _{x \geq 0} f(x) x^{k}<\infty, \quad \forall k \in \mathbf{N} .
$$

The seminorms on $\mathcal{L}^{+}(\mathcal{D})$ are labeled by the functions of the set $\mathcal{C}$ and by the natural numbers $\boldsymbol{N}$. Therefore \|\|$^{f, k}$ is a seminorm of the topology $\tau$ if and only if $(f, k)$ belongs to the set $\mathcal{C}_{N}:=\{(\mathcal{C}, \boldsymbol{N})\}$. We have

$$
X \in \mathcal{L}^{+}(\mathcal{D}) \rightarrow\|X\|^{f, k} \equiv \max \left\{\left\|f(N) X N^{k}\right\|,\left\|N^{k} X f(N)\right\|\right\}, \quad(f, k) \in \mathcal{C}_{N} .
$$

Here \|\| is the usual norm in $B(\mathcal{H})$. From this definition it follows that $\|X\|^{f, k}=\left\|X^{\dagger}\right\|^{f, k}$. In [6] it has also been proved that $\mathcal{L}^{+}(\mathcal{D})[\tau]$ is a complete locally convex topological *-algebra. 
Let us remark now that the two contributions in the definition (1.4) have exactly the same form. It is clear that, therefore, the estimate of $\left\|f(N) X N^{k}\right\|$ is quite close to that of $\left\|N^{k} X f(N)\right\|$, for any given $X \in \mathcal{L}^{+}(\mathcal{D})$. This is why we will identify $\|X\|^{f, k}$ with $\left\|f(N) X N^{k}\right\|$ in the following.

Moreover, using the spectral decomposition for $N, N=\sum_{l=0}^{\infty} l \Pi_{l}$, the seminorm $\|X\|^{f, k}$ can be written as follows, [6]:

$$
X \in \mathcal{L}^{+}(\mathcal{D}) \longrightarrow\|X\|^{f, k}=\sum_{l, s=0}^{\infty} f(l) s^{k}\left\|\Pi_{l} X \Pi_{s}\right\| .
$$

The paper is organized as follows:

in the next section we introduce the notion of weak $\tau$ strict contractions and discuss the existence (and the uniqueness) of a fixed point for these maps; in Section 3 we discuss the case in which the generalized contractions depend continuously on a parameter; Section 4 is devoted to examples and applications to differential equations, to ordinary quantum mechanics and to $\mathrm{QM}_{\infty}$. The outcome is contained in Section 5. In the Appendix we will introduce, for practical convenience, a different topology $\tau_{0}$, equivalent to $\tau$ and prove the non triviality of our construction. Of course $\mathcal{L}^{+}(\mathcal{D})\left[\tau_{0}\right]$ is again a complete locally convex topological ${ }^{*}$-algebra.

\section{$\S 2 . \quad$ The Weak $\tau$-Strict Contractions}

Let $\mathcal{B}$ be a $\tau$-complete subspace of $\mathcal{L}^{+}(\mathcal{D})$ and $T$ a map from $\mathcal{B}$ into $\mathcal{B}$. We say that $T$ is a weak $\tau$ strict contraction over $\mathcal{B}$, briefly $\mathrm{w} \tau \operatorname{sc}(\mathcal{B})$, if there exists a constant $c \in] 0,1\left[\right.$ such that, for all $(h, k) \in \mathcal{C}_{N}$, it exists a pair $\left(h^{\prime}, k^{\prime}\right) \in \mathcal{C}_{N}$ satisfying

$$
\|T x-T y\|^{h, k} \leq c\|x-y\|^{h^{\prime}, k^{\prime}} \quad \forall x, y \in \mathcal{B} .
$$

As in the standard situation, see [1], [2], [3], this definition does not imply that $\|T z\|^{h, k} \leq c\|z\|^{h^{\prime}, k^{\prime}}$ for all $z \in \mathcal{B}$ since $T$ is not a linear map in general. Of course, because of this lack of linearity, $T 0$ could be different from 0 ; however, any such $T$ defines in a natural way another map $T^{\prime}$ which is still a $\mathrm{w} \tau \operatorname{sc}(\mathcal{B})$ corresponding to the same quantities $c, h^{\prime}$ and $k^{\prime}$ as the original map $T$ and which satisfies $T^{\prime} 0=0$. In fact, let us put $T^{\prime} x:=T x-T 0$, for all $x \in \mathcal{B}$. Obviously we have $T^{\prime} 0=0$, and $\left\|T^{\prime} x-T^{\prime} y\right\|^{h, k}=\|T x-T y\|^{h, k} \leq c\|x-y\|^{h^{\prime}, k^{\prime}}$ for all choices of $x, y \in \mathcal{B}$. If $T 0=0$, equation (2.1) implies that

$$
\|T x\|^{h, k} \leq c\|x\|^{h^{\prime}, k^{\prime}} \quad \forall x \in \mathcal{B} .
$$


In what follows we will consider equations of the form $T x=x, T$ being a $\mathrm{w} \tau \operatorname{sc}(\mathcal{B})$. The first step consists in introducing the following subset of $\mathcal{B}$ :

$$
\mathcal{B}_{L} \equiv\left\{x \in \mathcal{B}: \sup _{(h, k) \in \mathcal{C}_{N}}\|T x-x\|^{h, k} \leq L\right\},
$$

$L$ being a fixed positive real number. It is clear that, if $L^{\prime}>L$, then $\mathcal{B}_{L} \subset \mathcal{B}_{L^{\prime}}$. Some of the properties of these sets are contained in the following

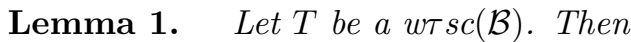

(a) if $T 0=0$ then any $x \in \mathcal{B}$ such that $\sup _{(h, k) \in \mathcal{C}_{N}}\|x\|^{h, k} \leq L_{1}$ belongs to $\mathcal{B}_{L}$ for $L \geq L_{1}(1+c)$;

(b) if $\|T 0\|^{h, k} \leq L_{2}$ for all $(h, k) \in \mathcal{C}_{N}$, then any $x \in \mathcal{B}$ such that $\sup _{(h, k) \in \mathcal{C}_{N}}\|x\|^{h, k} \leq L_{1}$ belongs to $\mathcal{B}_{L}$ for $L \geq L_{1}(1+c)+L_{2}$;

(c) if $x \in \mathcal{B}_{L}$ then $T^{n} x \in \mathcal{B}_{L}$, for all $n \in \mathbb{N}$;

(d) $\mathcal{B}_{L}$ is $\tau$-complete;

(e) if $\mathcal{B}_{L}$ is not empty, then $T$ is a $w \tau \operatorname{sc}\left(\mathcal{B}_{L}\right)$.

Proof. (a) Due to the hypothesis on $\|x\|^{h, k}$ and to equation (2.2) we have

$$
\begin{aligned}
\|T x-x\|^{h, k} & \leq\|T x\|^{h, k}+\|x\|^{h, k} \leq c\|x\|^{h^{\prime}, k^{\prime}}+\|x\|^{h, k} \\
& \leq c \sup _{\left(h^{\prime}, k^{\prime}\right) \in \mathcal{C}_{N}}\|x\|^{h^{\prime}, k^{\prime}}+\sup _{(h, k) \in \mathcal{C}_{N}}\|x\|^{h, k} \leq L_{1}(1+c) .
\end{aligned}
$$

(b) The proof uses the inequality

$$
\|T x-x\|^{h, k} \leq\|T x-T 0\|^{h, k}+\|T 0-x\|^{h, k},
$$

together with (2.1) for $y=0$ and the bound on $\|T 0\|^{h, k}$.

(c) We prove the statement by induction. For $n=1$ we have

$$
\|T(T x)-T x\|^{h, k} \leq c\|T x-x\|^{h^{\prime}, k^{\prime}} \leq c \sup _{\left(h^{\prime}, k^{\prime}\right) \in \mathcal{C}_{N}}\|T x-x\|^{h^{\prime}, k^{\prime}} \leq c L \leq L,
$$

Taking the $\sup _{(h, k) \in \mathcal{C}_{N}}$ of this inequality we conclude that $T x \in \mathcal{B}_{L}$. The second step of the induction goes as follows:

$$
\begin{aligned}
\left\|T\left(T^{n+1} x\right)-T^{n+1} x\right\|^{h, k} & =\left\|T\left(T^{n+1} x\right)-T\left(T^{n} x\right)\right\|^{h, k} \leq c\left\|T^{n+1} x-T^{n} x\right\|^{h^{\prime}, k^{\prime}} \\
& \leq c \sup _{\left(h^{\prime}, k^{\prime}\right) \in \mathcal{C}_{N}}\left\|T\left(T^{n} x\right)-T^{n} x\right\|^{h^{\prime}, k^{\prime}} \leq c L \leq L,
\end{aligned}
$$

which implies that $T^{n+1} x$ belongs to $\mathcal{B}_{L}$ whenever $T^{n} x$ does. 
(d) We will consider here the case in which $\mathcal{B}_{L}$ is non empty. Since $\mathcal{B}_{L}$ is a subset of a $\tau$-complete set, it is enough to check that $\mathcal{B}_{L}$ is $\tau$-closed. Let us take a sequence $\left\{x_{n}\right\} \in \mathcal{B}_{L}, \tau$-converging to an element $x$. We have to prove that $x \in \mathcal{B}_{L}$.

First of all, it is evident that $T$ is $\tau$-continuous: in fact, if $\left\{z_{n}\right\}$ is $\tau$ convergent to $z$, then $\left\{T z_{n}\right\} \tau$-converges to $T z$. Moreover, since $x_{n}$ belongs to $\mathcal{B}_{L}$ for all $n$, we have $\sup _{(h, k) \in \mathcal{C}_{N}}\left\|T x_{n}-x_{n}\right\|^{h, k} \leq L$ independently of $n$. We can conclude, therefore, that

$$
\|T x-x\|^{h, k} \leq \lim _{n \rightarrow \infty}\left\|T x_{n}-x_{n}\right\|^{h, k} \leq L
$$

which concludes the proof.

(e) This statement follows from the facts that $T$ is a $\mathrm{w} \tau \operatorname{sc}(\mathcal{B})$, that $T$ maps $\mathcal{B}_{L}$ into itself, and from the $\tau$-completeness of $\mathcal{B}_{L}$.

A consequence of this lemma is that, if $\mathcal{B}_{L}$ contains a single element, then $\mathcal{B}_{L}$ is rather a rich set. What the lemma does not say, is whether or not $\mathcal{B}_{L}$ contains at least one element. Of course, due to its definition, the answer will depend on the explicit form of the map $T$ and from the family of seminorms which define the topology. The non-triviality of the definition (2.3) is proved in the Appendix.

We give now our main fixed-point result for a $\mathrm{w} \tau \mathrm{sc}$.

Proposition 2. Let $T$ be a $w \tau \operatorname{sc}(\mathcal{B})$. Then

(a) $\forall x_{0} \in \mathcal{B}_{L}$ the sequence $\left\{x_{n} \equiv T^{n} x_{0}\right\}_{n \geq 0}$ is $\tau$-Cauchy in $\mathcal{B}_{L}$. Its $\tau$-limit, $x \in \mathcal{B}_{L}$, is a fixed point of $T$;

(b) if $x_{0}, y_{0} \in \mathcal{B}_{L}$ satisfy the condition $\sup _{(h, k) \in \mathcal{C}_{N}}\left\|x_{0}-y_{0}\right\|^{h, k}<\infty$, then $\tau-\lim _{n} T^{n} x_{0}=\tau-\lim _{n} T^{n} y_{0}$.

Proof. (a) First we observe that, due to the definition of $\mathcal{B}_{L}$, we have

$$
\begin{aligned}
\left\|x_{n+1}-x_{n}\right\|^{h, k} & =\left\|T x_{n}-T x_{n-1}\right\|^{h, k} \\
& \leq c\left\|x_{n}-x_{n-1}\right\|^{h_{1}, k_{1}} \leq \cdots \\
& \leq c^{n}\left\|T x_{0}-x_{0}\right\|^{h_{n}, k_{n}} \leq L c^{n},
\end{aligned}
$$

which implies, for any $n>m$,

$$
\left\|x_{n+1}-x_{m}\right\|^{h, k} \leq L \frac{c^{m}}{1-c}
$$

which goes to zero for $m$ (and $n$ ) diverging. Therefore the sequence $\left\{x_{n}\right\}_{n \geq 0}$ is $\tau$-Cauchy. Since $\mathcal{B}_{L}$ is $\tau$-complete, see Lemma 1 , there exists an element 
$x \in \mathcal{B}_{L}$ such that $x=\tau-\lim _{n} T^{n} x_{0}$. Now, the $\tau$-continuity of $T$ implies that $x$ is a fixed point. In fact:

$$
T x=T\left(\tau-\lim _{n} T^{n} x_{0}\right)=\tau-\lim _{n} T^{n+1} x_{0}=\tau-\lim _{n} x_{n+1}=x .
$$

(b) let us call $x=\tau-\lim _{n} T^{n} x_{0}$ and $y=\tau-\lim _{n} T^{n} y_{0}$. Then, using $n$ times inequality (2.1), we get

$$
\begin{aligned}
\|x-y\|^{h, k}=\lim _{n}\left\|T^{n} x_{0}-T^{n} y_{0}\right\|^{h, k} & \leq \lim _{n} c^{n}\left\|x_{0}-y_{0}\right\|^{h_{n}, k_{n}} \\
& \leq\left[\sup _{(h, k) \in \mathcal{C}_{N}}\left\|x_{0}-y_{0}\right\|^{h, k}\right] \lim _{n} c^{n}=0,
\end{aligned}
$$

for all seminorms. Therefore $x=y$.

Remarks. 1) The first remark is that if $x_{0}$ and $y_{0}$ are two operators of $\mathcal{L}^{+}(\mathcal{D})$ satisfying the bounds $\sup _{(h, k) \in \mathcal{C}_{N}}\left\|x_{0}\right\|^{h, k}=L_{x_{0}}$ and $\sup _{(h, k) \in \mathcal{C}_{N}}\left\|y_{0}\right\|^{h, k}$ $=L_{y_{0}}$ then, if $T 0=0$, both $x_{0}$ and $y_{0}$ belong to $\mathcal{B}_{L}$ for $L=\max \left(L_{x_{0}}(1+\right.$ c), $\left.L_{y_{0}}(1+c)\right)$ as a consequence of Lemma 1 . Moreover, it is easy to check that $x_{0}$ and $y_{0}$ satisfy the condition in (b) of the proposition, and, for this reason, they produce the same fixed point. To this same conclusion we arrive even if $T 0 \neq 0$ but $\|T 0\|^{h, k} \leq L_{2}$ for a positive constant $L_{2}$, independent of $(h, k)$.

2) The second remark concerns the non uniqueness of the fixed point given by our procedure. In fact, the statement (b) above implies uniqueness only within a certain class of possible fixed points, those obtained starting from elements of $\mathcal{B}_{L}$. It is useless to stress that other possibilities could exist for finding completely different fixed points which are not considered here. However, there exists a simple situation in which uniqueness is also ensured: it happens when the map $T$ is a $\tau$ strict contraction over $B$. Such a map differs from a $\mathrm{w} \tau \operatorname{sc}(\mathcal{B})$ in that the new seminorm \|\|$^{h^{\prime}, k^{\prime}}$ in the r.h.s. of inequality (2.1) coincides with the original one:

$\exists c \in] 0,1\left[\right.$ such that $\|T x-T y\|^{h, k} \leq c\|x-y\|^{h, k} \forall x, y \in \mathcal{B}$ and $\forall(h, k) \in \mathcal{C}_{N}$.

For such a contraction everything is much easier since a standard result, see [2], Theorem V.18, can be adapted here without major changes, and gives the existence and uniqueness of the fixed point.

3) The fact that the fixed point of the map $T$ belongs to $\mathcal{B}_{L}$ should not be a big surprise. As a matter of fact, this is true just because of the definition of fixed point. In fact, since $T x-x=0$, it is clear that $\sup _{(h, k) \in \mathcal{C}_{N}}\|T x-x\|^{h, k}=0$, which implies that $x \in \mathcal{B}_{L}$. 
4) This fixed point result is different from the one given in [2], Theorem 5.18 , where the sequence $\left\{T^{n} x\right\}$ could be constructed starting by any element $x$ of the complete metric space on which $T$ acts. Here, on the other way, the role of $\mathcal{B}_{L}$, as the set of the starting points for the sequences $\left\{x_{n}\right\}_{n \geq 0}$ producing the fixed points, is crucial!

5) We also remark that the hypothesis of Proposition 2, point (b), is verified by the pair $\left(x_{0}, y_{0} \equiv T^{m} x_{0}\right), m$ being a fixed natural, so that the related fixed points coincide. This is, again, not surprising since, of course, the two sequences $\left\{T^{n} x_{0}\right\}_{n \geq 0}$ and $\left\{T^{n}\left(T^{m} x_{0}\right)\right\}_{n \geq 0}$ must converge to the same element.

6) The same procedure can be generalized to the situation in which $\mathcal{L}^{+}(\mathcal{D})$ is replaced by an algebra (or a ${ }^{*}$-algebra, if needed) $\mathcal{A}$, which is complete with respect to a locally convex topology $\sigma$ defined by a (non countable) family of seminorms $p_{\alpha}$. In this case, if $\mathcal{B}$ is a $\sigma$-complete subspace of $\mathcal{A}$ and $T$ is a map from $\mathcal{B}$ into $\mathcal{B}$ we say that $T$ is a $\operatorname{w} \sigma \operatorname{sc}(\mathcal{B})$ if there exists a constant $c \in] 0,1[$ such that, for any seminorm $p_{\alpha}$, there exists a (different) seminorm $p_{\beta}$ for which

$$
p_{\alpha}(T x-T y) \leq c p_{\beta}(x-y), \quad \forall x, y \in \mathcal{B} .
$$

Lemma 1 and Proposition 2 can be stated with only minor changes.

\section{$\S 3 . \quad$ Continuity of Weak $\tau$-Strict Contractions}

In this section we consider the case in which the $\mathrm{w} \tau \operatorname{sc}(\mathcal{B})$ depends on a (real) parameter assuming that some kind of continuity holds. Besides its mathematical interest, this situation has a certain relevance in quantum mechanics, which will be discussed in the next section.

Let $I \subset \mathbb{R}$ be a set such that 0 is one of its accumulation points. A family of weak $\tau$ strict contractions $\left\{T_{\alpha}\right\}_{\alpha \in I}$ is said uniform if

1) $T_{\alpha}: \mathcal{B} \rightarrow \mathcal{B} \forall \alpha \in I, \mathcal{B}$ being a $\tau$-complete subspace of $\mathcal{L}^{+}(\mathcal{D})$;

2) $\forall(h, k) \in \mathcal{C}_{N}$ and $\forall \alpha \in I$ there exist $\left(h^{\prime}, k^{\prime}\right) \in \mathcal{C}_{N}$, independent of $\alpha$, and $\left.c_{\alpha} \in\right] 0,1[$, independent of $(h, k)$, such that

$$
\left\|T_{\alpha} x-T_{\alpha} y\right\|^{h, k} \leq c_{\alpha}\|x-y\|^{h^{\prime}, k^{\prime}}, \quad \forall x, y \in \mathcal{B} ;
$$

3) $\left.c_{-} \equiv \lim _{\alpha, 0} c_{\alpha} \in\right] 0,1[$.

Again, it is worthwhile to remark that none of the $T_{\alpha}$ is supposed to be linear, so that $T_{\alpha} x-T_{\alpha} y$ needs not to coincide with $T_{\alpha}(x-y)$.

An important consequence of this uniformity is that the element $\left(h_{n}, k_{n}\right) \in$ $\mathcal{C}_{N}$ in the r.h.s. of the inequality below is independent of the order of the maps $T_{\alpha}$ and only depends on the initial pair $(h, k)$ and on the number of maps, $n$ :

$$
\left\|T_{\alpha_{1}} T_{\alpha_{2}} \cdots T_{\alpha_{n}} x\right\|^{h, k} \leq c_{\alpha_{1}} c_{\alpha_{2}} \cdots c_{\alpha_{n}}\|x\|^{h_{n}, k_{n}} .
$$


We further say that the family $\left\{T_{\alpha}\right\}_{\alpha \in I}$ is $\tau$-strong Cauchy if, for all $(h, k) \in \mathcal{C}_{N}$ and $\forall y \in \mathcal{B}$,

$$
\left\|T_{\alpha} y-T_{\beta} y\right\|^{h, k} \rightarrow 0,
$$

whenever both $\alpha$ and $\beta$ go to zero.

With natural notation, we call $\mathcal{B}_{L}^{(\alpha)}$ the set $\mathcal{B}_{L}$ related to the map $T_{\alpha}$,

$$
\mathcal{B}_{L}^{(\alpha)} \equiv\left\{x \in \mathcal{B}: \sup _{(h, k) \in \mathcal{C}_{N}}\left\|T_{\alpha} x-x\right\|^{h, k} \leq L\right\} .
$$

We stress that, even if the set $\mathcal{B}$ is unique for all the maps $T_{\alpha}$, the sets $\mathcal{B}_{L}^{(\alpha)}$ may differ from each other.

Proposition 3. Let $\left\{T_{\alpha}\right\}_{\alpha \in I}$ be a $\tau$-strong Cauchy uniform family of $w \tau \operatorname{sc}(\mathcal{B})$. Then

1) There exists a $w \tau \operatorname{sc}(\mathcal{B}), T$, which satisfies the following relations :

$$
\left\|T y-T_{\alpha} y\right\|^{h, k} \rightarrow 0 \quad \forall y \in \mathcal{B}, \quad \forall(h, k) \in \mathcal{C}_{N}
$$

and

$$
\|T y-T z\|^{h, k} \leq c_{-}\|y-z\|^{h^{\prime}, k^{\prime}} \quad \forall y, z \in \mathcal{B},
$$

where $\left(h^{\prime}, k^{\prime}\right)$ are those of inequality (3.1).

2) let $\left\{x_{\alpha}\right\}_{\alpha \in I}$ be a family of fixed points of the net $\left\{T_{\alpha}\right\}_{\alpha \in I}: T_{\alpha} x_{\alpha}=x_{\alpha}$, $\forall \alpha \in I$. If $\left\{x_{\alpha}\right\}_{\alpha \in I}$ is a $\tau$-Cauchy net then, calling $x$ its $\tau$-limit in $\mathcal{B}, x$ is a fixed point of $T$.

3) If the set $\cap_{\alpha \in I} \mathcal{B}_{L}^{(\alpha)}$ is not empty and if the following commutation rule holds

$$
T_{\alpha}\left(T_{\beta} y\right)=T_{\beta}\left(T_{\alpha} y\right), \quad \forall \alpha, \beta \in I \text { and } \forall y \in \mathcal{B},
$$

then, calling

$$
x_{\alpha}=\tau-\lim _{n \rightarrow \infty} T_{\alpha}^{n} x^{0} \quad x^{0} \in \cap_{\alpha \in I} \mathcal{B}_{L}^{(\alpha)},
$$

each $x_{\alpha}$ is a fixed point of $T_{\alpha}, T_{\alpha} x_{\alpha}=x_{\alpha}$ and $\left\{x_{\alpha}\right\}_{\alpha \in I}$ is a $\tau$-Cauchy net. Moreover $\tau-\lim _{\alpha \rightarrow 0} x_{\alpha}$ is a fixed point of $T$.

Proof.

1) Since $\mathcal{B}$ is $\tau$-complete and since $\left\{T_{\alpha}\right\}_{\alpha \in I}$ is $\tau$-strong Cauchy, for any $y \in \mathcal{B}$ there exists an element $z \in \mathcal{B}$ such that $z=\tau-\lim _{\alpha, 0} T_{\alpha} y$. We use $z$ to define $T$ as

$$
T y:=z .
$$


It is evident that $T$ maps $\mathcal{B}$ into itself and that $\left\|T y-T_{\alpha} y\right\|^{h, k} \rightarrow 0$ for every $y \in \mathcal{B}$ and for all $(h, k) \in \mathcal{C}_{N}$.

Equation (3.5) follows from:

$$
\|T y-T z\|^{h, k}=\lim _{\alpha \rightarrow 0}\left\|T_{\alpha} y-T_{\alpha} z\right\|^{h, k} \leq \lim _{\alpha \rightarrow 0} c_{\alpha}\|y-z\|^{h^{\prime}, k^{\prime}}=c_{-}\|y-z\|^{h^{\prime}, k^{\prime}},
$$

for all $y, z \in \mathcal{B}$.

2) Since $\left\{x_{\alpha}\right\}_{\alpha \in I}$ is $\tau$-Cauchy, there exists in $\mathcal{B}$ an element $x=\tau-\lim _{\alpha} x_{\alpha}$. We use the equality $T_{\alpha} x_{\alpha}=x_{\alpha}$ to prove that $T x=x$. In fact

$$
\|T x-x\|^{h, k} \leq\left\|T x-T_{\alpha} x\right\|^{h, k}+\left\|T_{\alpha} x-x_{\alpha}\right\|^{h, k}+\left\|x_{\alpha}-x\right\|^{h, k} \rightarrow 0 .
$$

This is because all the contributions in the rhs goes to zero for $\alpha$ going to zero: the first because of the equation (3.4), the third because of the definition of $x$ and the second for the same reason, since

$$
\left\|T_{\alpha} x-x_{\alpha}\right\|^{h, k}=\left\|T_{\alpha} x-T_{\alpha} x_{\alpha}\right\|^{h, k} \leq c_{\alpha}\left\|x-x_{\alpha}\right\|^{h, k} .
$$

3) Since it exists an element $x^{0} \in \cap_{\alpha \in I} \mathcal{B}_{L}^{(\alpha)}$, Proposition 2 implies that $x_{\alpha}=\tau-\lim _{n \rightarrow \infty} T_{\alpha}^{n} x^{0}$ is a fixed point of $T_{\alpha}$, and $x_{\beta}=\tau-\lim _{n \rightarrow \infty} T_{\beta}^{n} x^{0}$ is a fixed point of $T_{\beta}$. This means, using the definition of the limit, that for any fixed $\epsilon>0$ and for each $(h, k) \in \mathcal{C}_{N}$, there exists an integer $m$ such that

$$
\left\|T_{\alpha}^{m} x^{0}-x_{\alpha}\right\|^{h, k}+\left\|T_{\beta}^{m} x^{0}-x_{\beta}\right\|^{h, k} \leq \frac{2 \epsilon}{3} .
$$

For this fixed $m$ we now estimate $\left\|T_{\alpha}^{m} x^{0}-T_{\beta}^{m} x^{0}\right\|^{h, k}$. It is possible to show that the following inequality holds:

$$
\left\|T_{\alpha}^{m} x^{0}-T_{\beta}^{m} x^{0}\right\|^{h, k} \leq m\left\|T_{\alpha} x^{0}-T_{\beta} x^{0}\right\|^{h_{m-1}, k_{m-1}}
$$

where $\left(h_{m-1}, k_{m-1}\right)$ only depends on the origin pair $(h, k)$ and on $m$, but not on $\alpha$ and $\beta$. We prove this inequality only for $m=2$. Its generalization to larger values of $m$ is straightforward.

$$
\begin{aligned}
\| T_{\alpha}^{2} x^{0} & -T_{\beta}^{2} x^{0}\left\|^{h, k} \leq\right\| T_{\alpha}\left(T_{\alpha} x^{0}\right)-T_{\alpha}\left(T_{\beta} x^{0}\right)\left\|^{h, k}+\right\| T_{\alpha}\left(T_{\beta} x^{0}\right)-T_{\beta}\left(T_{\beta} x^{0}\right) \|^{h, k} \\
& =\left\|T_{\alpha}\left(T_{\alpha} x^{0}\right)-T_{\alpha}\left(T_{\beta} x^{0}\right)\right\|^{h, k}+\left\|T_{\beta}\left(T_{\alpha} x^{0}\right)-T_{\beta}\left(T_{\beta} x^{0}\right)\right\|^{h, k} \\
& \leq\left(c_{\alpha}+c_{\beta}\right)\left\|T_{\alpha} x^{0}-T_{\beta} x^{0}\right\|^{h_{1}, k_{1}} \leq 2\left\|T_{\alpha} x^{0}-T_{\beta} x^{0}\right\|^{h_{1}, k_{1}} .
\end{aligned}
$$

Here we have used condition (3.6) together with the remark leading to inequality (3.2). 
Now we can collect all these results to prove the statement: let $m$ be the fixed integer introduced in equation (3.9). We have

$$
\begin{aligned}
\left\|x_{\alpha}-x_{\beta}\right\|^{h, k} & \leq\left\|x_{\alpha}-T_{\alpha}^{m} x^{0}\right\|^{h, k}+\left\|T_{\alpha}^{m} x^{0}-T_{\beta}^{m} x^{0}\right\|^{h, k}+\left\|T_{\beta}^{m} x^{0}-x_{\beta}\right\|^{h, k} \\
& \leq \frac{2 \epsilon}{3}+m\left\|T_{\alpha} x^{0}-T_{\beta} x^{0}\right\|^{h_{m-1}, k_{m-1}} .
\end{aligned}
$$

Since $\left\{T_{\alpha}\right\}_{\alpha \in I}$ is $\tau$-strong Cauchy then there exists a ball, $P(0, \gamma)$, centered in zero and with radius $\gamma$, which depends on $\left(h_{m-1}, k_{m-1}\right), \epsilon$ and $m$, such that, for all $\alpha$ and $\beta$ inside this ball, the inequality $\left\|T_{\alpha} x^{0}-T_{\beta} x^{0}\right\|^{h_{m-1}, k_{m-1}} \leq \epsilon / 3 m$ holds. In conclusion we have proved that

$$
\forall \epsilon>0, \forall(h, k) \in \mathcal{C}_{N} \exists P(0, \gamma) \text { such that }\left\|x_{\alpha}-x_{\beta}\right\|^{h, k} \leq \epsilon, \quad \forall \alpha, \beta \in P(0, \gamma) .
$$

This implies that $\left\{x_{\alpha}\right\}_{\alpha \in I}$ is a $\tau$-Cauchy net. The last statement finally follows from point 2).

We now consider a different kind of problem: let $\left\{T_{\alpha}\right\}_{\alpha \in I}$ be an uniform family of $\operatorname{w} \tau \operatorname{sc}(\mathcal{B}), \tau$-strong convergent to a $\mathrm{w} \tau \operatorname{sc}(\mathcal{B}), T$; let $x$ be a fixed point of $T$ and $x_{\alpha}$ a fixed point of $T_{\alpha}, \alpha \in I$. We wonder if the net $\left\{x_{\alpha}\right\}_{\alpha \in I}$ is $\tau$ converging to $x$. Of course, sic stantibus rebus, the answer cannot be positive, because of the non uniqueness of the fixed points of a generic $\mathrm{w} \tau \mathrm{sc}$. In order to say something more we must impose other conditions. We prove the following

Proposition 4. Let $\left\{T_{\alpha}\right\}_{\alpha \in I}$ be an uniform family of $w \tau s c(\mathcal{B}), \tau$-strong converging to the $w \tau s c(\mathcal{B}) T$ and satisfying condition (3.6).

If the set $\left(\cap_{\alpha \in I} \mathcal{B}_{L}^{(\alpha)}\right) \cap \mathcal{B}$ contains an element $x^{0}$ then, defining

$$
x_{\alpha}=\tau-\lim _{n \rightarrow \infty} T_{\alpha}^{n} x^{0} \quad \text { and } x=\tau-\lim _{n \rightarrow \infty} T^{n} x^{0}, \quad \alpha \in I,
$$

$\left\|x_{\alpha}-x\right\|^{h, k} \rightarrow 0$ for all $(h, k) \in \mathcal{C}_{N}$.

Proof. First of all we observe that a consequence of condition (3.6) is the analogous commutation rule for the maps $T_{\alpha}$ and $T$ :

$$
T_{\alpha}(T y)=T\left(T_{\alpha} y\right), \quad \forall \alpha \in I \text { and } \forall y \in \mathcal{B} .
$$

Using this result, the statement follows from the same argument as the one used in the proof of Proposition 3, point 3).

An easier result can be obtained under stronger assumptions. First we call $\left\{T_{\alpha}\right\}_{\alpha \in I}$ an uniform family of $\tau \operatorname{sc}(\mathcal{B})$ if it is a $\operatorname{w} \tau \operatorname{sc}(\mathcal{B})$ and if $\left(h^{\prime}, k^{\prime}\right)=(h, k)$, in inequality (3.1). For any fixed $\alpha$ we have already observed in Section 2 that the fixed point of $T_{\alpha}$ is unique. Here we have: 
Proposition 5. Let $\left\{T_{\alpha}\right\}_{\alpha \in I}$ be an uniform family of $\tau s c(\mathcal{B}), \tau$-strong converging to the $\tau \operatorname{sc}(\mathcal{B}) T$, and such that $c_{\alpha} \leq c^{+} \forall \alpha \in I$ with $c^{+}<1$.

If $\left\{x_{\alpha}\right\}_{\alpha \in I} \subset \mathcal{B}$ and $x \in \mathcal{B}$ are such that $T_{\alpha} x_{\alpha}=x_{\alpha}$ for all $\alpha \in I$ and $T x=x$, then $\left\|x_{\alpha}-x\right\|^{h, k} \rightarrow 0$ for all $(h, k) \in \mathcal{C}_{N}$.

Proof. Under these hypotheses we have

$$
\begin{aligned}
& \left\|x_{\alpha}-x\right\|^{h, k}=\left\|T_{\alpha} x_{\alpha}-T x\right\|^{h, k} \leq\left\|T_{\alpha} x_{\alpha}-T_{\alpha} x\right\|^{h, k}+\left\|T_{\alpha} x-T x\right\|^{h, k} \\
& \leq c_{\alpha}\left\|x_{\alpha}-x\right\|^{h, k}+\left\|T_{\alpha} x-T x\right\|^{h, k} \leq c^{+}\left\|x_{\alpha}-x\right\|^{h, k}+\left\|T_{\alpha} x-T x\right\|^{h, k} .
\end{aligned}
$$

Using the bound on $c^{+}$we conclude that $\left\|x_{\alpha}-x\right\|^{h, k} \leq \frac{1}{1-c^{+}}\left\|T_{\alpha} x-T x\right\|^{h, k}$, which goes to zero by assumption.

\section{$\S 4 . \quad$ Examples and Applications}

We start this section giving few examples of $\mathrm{w} \tau \mathrm{sc}$ (we omit the space $\mathcal{B}$ whenever this coincides with the whole $\mathcal{L}^{+}(\mathcal{D})$ ).

Using the same notations as in the Introduction, we define the following maps acting on $x \in \mathcal{L}^{+}(\mathcal{D})$ :

$$
T_{\alpha}^{(i, j)} x \equiv \alpha N^{i} x N^{j}
$$

and

$$
T_{l} x \equiv\left[N^{l}, x\right]=N^{l} x-x N^{l} .
$$

Here $\alpha$ is a complex number with modulus strictly less than 1 , while $i, j$ and $l$ are natural numbers. We also assume that $N^{-1}$ exists (as a bounded operator) and satisfies the bound $2\left\|N^{-1}\right\|^{l}<1$. Both $T_{\alpha}^{(i, j)}$ and $T_{l}$ are linear and it can be easily checked that they are $\mathrm{w} \tau \mathrm{sc}$. In fact, introducing the function $h_{i}(x)=$ $x^{i} h(x)$, which still belongs to the set $\mathcal{C}$, we get $\left\|T_{\alpha}^{(i, j)} x\right\|^{h, k}=|\alpha|\|x\|^{h_{i}, k+j}$ and $\left\|T_{l} x\right\|^{h, k} \leq 2\left\|N^{-1}\right\|^{l}\|x\|^{h_{l}, k+l}$. Our claim finally follows from the linearity of the maps.

More relevant is the following application to differential equations which shows that it is possible to associate a $\mathrm{w} \tau \mathrm{sc}$ to some differential equations over $\mathcal{L}^{+}(\mathcal{D})$.

Let $\delta$ be a positive real number, $\left\{d_{h, k}\right\}_{(h, k) \in \mathcal{C}_{N}}$ a net of positive real numbers and $x_{0}$ an element of the algebra $\mathcal{L}^{+}(\mathcal{D})$ (corresponding to the initial condition). Let us now introduce the following sets:

$$
I_{\delta} \equiv[0, \delta],
$$




$$
\begin{aligned}
L_{x_{0},\{d\}, \delta} & \equiv\left\{X \in \mathcal{L}^{+}(\mathcal{D}): \forall(h, k) \in \mathcal{C}_{N}\right. \\
\exists\left(h^{\prime}, k^{\prime}\right) & \left.\in \mathcal{C}_{N}:\left\|X-x_{0}\right\|^{h, k} \leq \delta d_{h^{\prime}, k^{\prime}}\right\},
\end{aligned}
$$

and, finally,

$$
\mathcal{F} \equiv I_{\delta} \times L_{x_{0},\{d\}, \delta} .
$$

It is clear that this set is not empty; in fact, among other elements, it contains $x_{0}$ for any values of $\delta$ and for any choice of the net $\{d\} \equiv\left\{d_{h, k}\right\}_{(h, k) \in \mathcal{C}_{N}}$. (In order to simplify the notation, we do not write the explicit dependence of $\mathcal{F}$ on $x_{0},\{d\}$ and $\delta$.)

We further introduce the following set of functions:

$$
\begin{gathered}
\mathcal{M} \equiv\left\{z(t): I_{\delta} \rightarrow \mathcal{L}^{+}(\mathcal{D}), \tau-\text { continuous and such that } \forall(h, k) \in \mathcal{C}_{N}\right. \\
\left.\exists\left(h^{\prime}, k^{\prime}\right) \in \mathcal{C}_{N}:\left\|z(t)-x_{0}\right\|^{h, k} \leq \delta d_{h^{\prime}, k^{\prime}}\right\} .
\end{gathered}
$$

Let now $f(t, x)$ be a function defined on $\mathcal{F}$ which takes values in $\mathcal{L}^{+}(\mathcal{D})$, and for which a constant $M$ exists, with $0<M<1 / \delta$, such that for all $(h, k) \in C_{N}$, there exist two pairs $\left(h^{\prime}, k^{\prime}\right),\left(h^{\prime \prime}, k^{\prime \prime}\right) \in C_{N}$ satisfying

$$
\begin{aligned}
\|f(t, x)\|^{h, k} & \leq d_{h^{\prime}, k^{\prime}} & \forall(t, x) & \in \mathcal{F} \\
\|f(t, x)-f(t, y)\|^{h, k} & \leq M\|x-y\|^{h^{\prime \prime}, k^{\prime \prime}} & \forall(t, x),(t, y) & \in \mathcal{F} .
\end{aligned}
$$

For such a function we consider the following differential equation

$$
\frac{d x(t)}{d t}=f(t, x(t)), \quad x(0)=x_{0},
$$

which can be written in integral form as

$$
x(t)=x_{0}+\int_{0}^{t} d s f(s, x(s)) .
$$

Let us now introduce the following map $U$ on $\mathcal{M}$ :

$$
(U z)(t) \equiv x_{0}+\int_{0}^{t} d s f(s, z(s))
$$

$t \in I_{\delta}$. It is obvious that, for a generic function $f(t, x)$, the map $U$ is not linear and $U 0 \neq 0$. It can be proven that $U$ is a $\operatorname{w} \tau_{\delta} \operatorname{sc}(\mathcal{M}), \mathcal{M}$ being endowed with the topology $\tau_{\delta}$ defined by the following seminorms:

$$
\|z\|_{\delta}^{h, k}:=\sup _{t \in I_{\delta}}\|z(t)\|^{h, k}
$$

The proof follows these lines: 
first of all, it is easy to check that $\mathcal{M}$ is $\tau_{\delta}$-closed;

secondly, due to the bound (4.5), we can verify that the function $(U z)(t)$ is $\tau$-continuous. In fact, since $z(s)$ belongs to $\mathcal{M}$, then $(s, z(s))$ belongs to $\mathcal{F}$. This implies that, for all $(h, k) \in \mathcal{C}_{N}$ there exists another pair $\left(h^{\prime}, k^{\prime}\right) \in \mathcal{C}_{N}$ such that

$$
\left\|(U z)(t)-(U z)\left(t_{0}\right)\right\|^{h, k} \leq \int_{t_{0}}^{t}\|f(s, z(s))\|^{h, k} d s \leq d_{h^{\prime}, k^{\prime}}\left|t-t_{0}\right| \rightarrow 0,
$$

when $t \rightarrow t_{0}$.

Yet, with analogous estimates, we also conclude that $\left\|(U z)(t)-x_{0}\right\|^{h, k} \leq$ $\delta d_{h^{\prime}, k^{\prime}}$ for all $t \in I_{\delta}$, and that, therefore, $U$ maps $\mathcal{M}$ into itself;

finally, condition (4.6) produces the following estimate

$$
\|U y-U z\|_{\delta}^{h, k} \leq M \delta\|y-z\|_{\delta}^{h^{\prime}, k^{\prime}}
$$

for all $y, z$ in $\mathcal{M}$. Therefore, since $M \delta<1, U$ is a ${ } \tau_{\delta} \operatorname{sc}(\mathcal{M})$.

Simple examples of functions satisfying conditions (4.5) and (4.6) are:

(a) $f_{1}(t, x)=\varphi(t) \mathbb{1}$, with $|\varphi(t)| \leq 1$ for all $t \in I_{\delta}$. For this example we fix the net $\{d\}$ as follows: $d_{h, k}:=\|\mathbb{1}\|^{h, k}$, while $x_{0}$ and $\delta$ are completely free;

(b) $f_{2}(t, x)=\varphi(t) X$, with $|\varphi(t)| \leq 1 / 2 \delta$ for all $t \in I_{\delta}$ and $X \in \mathcal{L}^{+}(\mathcal{D})$. Here we take for convenience $x_{0}=0$ while $\delta$ and $\{d\}$ are free;

(c) $f_{3}(t, x)=\varphi(t) N^{l} X$, with $|\varphi(t)| \leq 1 / 2 \delta$ for all $t \in I_{\delta}, l \in \boldsymbol{N}, X \in \mathcal{L}^{+}(\mathcal{D})$ and $N$ is the number operator introduced in Section 1. Again, we fix $x_{0}=0$, while $\delta$ and $\{d\}$ are free.

In order to apply Proposition 2 to the analysis of the differential equation (4.7) we first have to check that the set $\mathcal{B}_{L}$ is non-empty. In other words, it is necessary to check that there exists (at least) an element $z_{0}(t) \in \mathcal{M}$ such that

$$
\sup _{(h, k) \in \mathcal{C}_{N}}\left\|\left(U z_{0}\right)(t)-z_{0}(t)\right\|_{\delta}^{h, k} \leq L,
$$

for a fixed positive constant $L$.

In general this check is not easy. However, if the function $f(t, x)$ and the initial condition $x_{0}$ satisfy, for a given $L^{\prime}>0$, the estimate

$$
\left\|f\left(t, x_{0}\right)\right\|^{h, k} \leq L^{\prime}, \quad \forall t \in I_{\delta}, \forall(h, k) \in \mathcal{C}_{N},
$$

then we can conclude that condition (4.11) is verified by choosing $z_{0}(t) \equiv x_{0}$ for all $t \in I_{\delta}$. In fact, with this choice, we have

$$
\left\|\left(U z_{0}\right)(t)-z_{0}(t)\right\|_{\delta}^{h, k}=\sup _{t \in I_{\delta}}\left\|\int_{0}^{t} f\left(s, x_{0}\right) d s\right\|^{h, k} \leq \sup _{t \in I_{\delta}} \int_{0}^{t}\left\|f\left(s, x_{0}\right)\right\|^{h, k} d s \leq L^{\prime} \delta,
$$


which implies the bound (4.11). In other words, if condition (4.12) holds, $x_{0}$ can be considered as a good starting point to construct the solution of the differential equation.

A simple example in which condition (4.12) is satisfied is $f(t, x)=\varphi(t) x$. Here $\varphi(t)$ is a "regular" function and the initial condition $x_{0}$ is defined using the strategy used in the Appendix in the proof of the non triviality of the set $\mathcal{B}_{L}$.

In conclusion we have shown that, under some conditions on the function $f(t, x)$ (which are not very different from the ones usually required in connection with the Cauchy problem), the existence, but not the uniqueness, of the solution of the differential equation (4.7) follows from our results.

This procedure can be applied straightforwardly to a generic quantum mechanical dynamical problem.

Let $x$ be an element of the algebra $\mathcal{L}^{+}(\mathcal{D})$ related to some quantum mechanical problem whose time evolution we are interested in. For instance, we can think of $\mathcal{D}$ as the domain of all the powers of the number operator $N \equiv a^{\dagger} a$, $a$ and $a^{\dagger}$ being as in the Introduction. Let $H=N$ be the hamiltonian of the system, which will be used to construct the seminorms: $\|Y\|^{h, k}=\left\|h(H) Y H^{k}\right\|$. The time evolution $x(t)$ is driven by the following Heisenberg equation:

$$
\frac{d x(t)}{d t}=i[H, x(t)]
$$

with initial condition $x\left(t_{0}\right)=x_{0}$.

It is well known that a formal solution of this equation does exist, and that its form is $x(t)=e^{i H t} x_{0} e^{-i H t}$. Now we want to show that the existence of the solution of the equation (4.13) can also be obtained by using our analysis of abstract differential equations. In particular, we will prove that to the Heisenberg equation of motion for an observable $x$ can be associated a $\operatorname{w} \tau \operatorname{sc}(\mathcal{M})$.

Calling $f(t, x(t))=i[H, x(t)]$ we can write the differential equation (4.13) in the integral form

$$
x(t)=x_{0}+\int_{0}^{t} f(s, x(s)) d s .
$$

It may be worthwhile to notice that this is an example in which the function $f(t, z)$ is linear in $z$ and does not depend explicitly on $t$. We now check that the function $f(t, x(t))$ satisfies conditions (4.5) and (4.6). First of all, we define the net $\{d\}$ by $d_{h, k}:=\left\|x_{0}\right\|^{h, k}$. Secondly, fixed a $\delta>0$, we introduce the sets defined in (4.1)-(4.4). In the following we will assume also the following bound on $H^{-1}$ :

$$
\left\|H^{-1}\right\| \leq \frac{1}{2(\delta+1)}
$$


Needless to say, this is not a strong assumption since, in any case, a constant can be added to $H$ without affecting the equation of motion. We have, for $(t, z) \in \mathcal{F}$,

$$
\begin{aligned}
\|f(t, z)\|^{h, k} & =\left\|h(H) f(t, z) H^{k}\right\|=\left\|h(H)[H, z] H^{k}\right\| \\
& \leq\left\|h(H) H z H^{k}\right\|+\left\|h(H) z H^{k+1}\right\| \leq 2\left\|H^{-1}\right\|\|z\|^{h_{1}, k+1},
\end{aligned}
$$

where $h_{1}(x)=x h(x)$. Now we use the fact that $z$ belongs to $L_{x_{0},\{d\}, \delta}$. This implies that, for all $(h, k) \in \mathcal{C}_{N}$, it exists another element in $\mathcal{C}_{N},\left(h^{\prime}, k^{\prime}\right)$, such that $\left\|z-x_{0}\right\|^{h, k} \leq \delta d_{h^{\prime}, k^{\prime}}$. Therefore $\|z\|^{h_{1}, k+1}$ can be estimated by $\delta d_{h_{1}^{\prime},(k+1)^{\prime}}+$ $d_{h_{1}, k+1}$. Moreover, defining $\left(h^{\prime}, k^{\prime}\right)$ as $\left(h_{1}^{\prime},(k+1)^{\prime}\right)$ if $d_{h_{1}^{\prime},(k+1)^{\prime}} \geq d_{h_{1}, k+1}$ and as $\left(h_{1}, k+1\right)$ otherwise, we conclude that $\|z\|^{h_{1}, k+1} \leq(\delta+1) d_{h^{\prime}, k^{\prime}}$. Therefore, recalling the bound on $\left\|H^{-1}\right\|$, we find that

$$
\|f(t, z)\|^{h, k} \leq d_{h^{\prime}, k^{\prime}}
$$

whenever $(t, z) \in \mathcal{F}$, which is exactly condition (4.5).

Moreover, for any $(t, z)$ and $(t, y)$ in $\mathcal{F}$,

$$
\begin{aligned}
\|f(t, x)-f(t, y)\|^{h, k} & \leq\left\|h(H) H(x-y) H^{k}\right\|+\left\|h(H)(x-y) H^{k+1}\right\| \\
& \leq 2\left\|H^{-1}\right\| \mid h_{1}(H)(x-y) H^{k+1} \| \\
& =2\left\|H^{-1}\right\|\|x-y\|^{h_{1}, k+1} \leq \frac{1}{1+\delta}\|x-y\|^{h_{1}, k+1} .
\end{aligned}
$$

We conclude that both conditions on the function $f(t, x)$ are satisfied since $M \equiv 1 /(1+\delta)<1 / \delta$, so that the map $U$ related to $H$ as in $(4.9)$ is a $\mathrm{w} \tau \operatorname{sc}(\mathcal{M})$.

For what concerns the starting element which produces the fixed point, the situation is again very close to that of general differential equations: if our initial condition $x_{0}$ satisfies the bound $\left\|x_{0}\right\|^{h, k} \leq m$ for all $(h, k) \in \mathcal{C}_{N}$, then we can check that the choice $z_{0}(t)=x_{0}$ for all $t \in I_{\delta}$ produces an element of the set $\mathcal{M}_{L}:=\left\{y(t) \in \mathcal{M}: \sup _{(h, k) \in \mathcal{C}_{N}}\|U y-y\|_{\delta}^{h, k} \leq L\right\}$, for $L=m \delta / 1+\delta$, which can be used to construct the sequence $\left\{U^{n} x_{0}\right\}_{n \in N} \tau_{\delta}$-converging to the solution of the Heisenberg equation.

We end this section with another physical application, consequence of the results discussed in Section 3. We want to stress that now the philosophy is rather different from that of the previous application where an existence result for the Heisenberg equation of motion was deduced. Here, on the other hand, we want to find the time evolution for a $Q M_{\infty}$ system in the thermodynamical limit.

Let us consider a physical system whose energy is given by a certain unbounded self-adjoint operator $H$, densely defined and invertible. We define $\mathcal{D}$ 
to be the domain of all the powers of the operator $H$, and $\mathcal{L}^{+}(\mathcal{D})[\tau]$ the topological *-algebra given in the Introduction. The seminorms are the usual ones, $\|X\|^{h, k}=\left\|h(H) X H^{k}\right\|,(h, k) \in \mathcal{C}_{N}$. As widely discussed in the literature, the rigorous approach to the physical model implies, as a first step, the introduction of a cut-off $\alpha$ which makes it well defined, and the related hamiltonian $H_{\alpha}$ a self-adjoint bounded operator. In what follows we will assume that $\alpha$ takes value in a given subset $I$ of $\mathbb{R}$ and that the limit $\alpha \rightarrow 0$ corresponds to the removal of the cutoff. Moreover we will assume that $H$ and $H_{\alpha}$ satisfy the following properties, for a given $\delta>0$ :

$$
\left[H_{\alpha}, H_{\beta}\right]=0, \quad \forall \alpha, \beta \in I
$$

$$
c_{\alpha} \equiv 2 \delta\left\|H^{-1}\right\|\left\|H^{-1} H_{\alpha}\right\|<1, \quad \forall \alpha \in I
$$

and

$$
\lim _{\alpha \rightarrow 0} 2\left\|H^{-1}\right\|\left\|H^{-1} H_{\alpha}\right\|>0
$$

$$
\lim _{\alpha \rightarrow 0}\left\|\left[H-H_{\alpha}, Y\right]\right\|^{h, k}=0, \quad \forall Y \in \mathcal{L}^{+}(\mathcal{D})
$$

$$
\left\|H^{-1}\right\| \leq \frac{1}{2(\delta+1)} .
$$

Before going on, we remark that conditions (p1) and (p3) together imply that

$$
\left[H, H_{\alpha}\right]=0, \quad \forall \alpha \in I .
$$

As discussed before, a typical problem in $\mathrm{QM}_{\infty}$, consists in solving first the Heisenberg equations of motion

$$
\frac{x_{\alpha}(t)}{d t}=i\left[H_{\alpha}, x_{\alpha}(t)\right], \quad \text { with } x_{\alpha}(0)=x
$$

for a general observable $x$ in $\mathcal{L}^{+}(\mathcal{D})[\tau]$, and, as a second step, trying to remove the cutoff $\alpha$. This is equivalent to find the $\tau$-limit of $x_{\alpha}(t)$ for $\alpha$ going to zero. In this way we obtain the dynamics of the model and the time evolution of $x$, $x(t):=\tau-\lim _{\alpha} x_{\alpha}(t)$.

As we know, the solution of equation (4.20) is, for finite $\alpha$,

$$
x_{\alpha}(t)=\exp \left(i H_{\alpha} t\right) x \exp \left(-i H_{\alpha} t\right) \text {. }
$$


Quite often, this expression is of little use since removing the cutoff in (4.21) is much harder than working with the integral version of equation (4.20):

$$
x_{\alpha}(t)=x+i \int_{0}^{t} d s\left[H_{\alpha}, x_{\alpha}(s)\right] .
$$

To analyze the removal of the cutoff, we first define

$$
F_{\alpha}\left(x_{\eta}(s)\right) \equiv i\left[H_{\alpha}, x_{\eta}(s)\right], \quad \alpha, \eta \in I .
$$

Due to equations (4.14) and (4.21), we have:

$$
\begin{gathered}
F_{\alpha}\left(y_{\eta}(s)\right)=\exp \left(i H_{\eta} s\right) F_{\alpha}(y) \exp \left(-i H_{\eta} s\right) \\
\forall \alpha, \eta \in I \text { and } \forall y \in \mathcal{L}^{+}(\mathcal{D}) .
\end{gathered}
$$

It is convenient to introduce the set $\mathcal{L}_{\gamma}^{+}(\mathcal{D})$ defined as follows: we fix a value $\gamma$ in the set $I ; \mathcal{L}_{\gamma}^{+}(\mathcal{D})$ is the set of all the elements $y \in \mathcal{L}^{+}(\mathcal{D})$ such that an element $y_{0} \in \mathcal{L}^{+}(\mathcal{D})$ exists which satisfies $y=\exp \left(i H_{\gamma} t\right) y_{0} \exp \left(-i H_{\gamma} t\right)$. Here both $y$ and $y_{0}$ could depend on time. Obviously, using equation (4.19), it is easily checked that $\mathcal{L}_{\gamma}^{+}(\mathcal{D})$ is again $\tau$-complete. Moreover, it is also clear that $\mathcal{L}_{\gamma}^{+}(\mathcal{D})$ does not differ significantly from $\mathcal{L}^{+}(\mathcal{D})$ even from a purely algebraical point of view. As a matter of fact, its introduction is clearly only a technicality. We define on this set the following map $U_{\alpha}$ :

$$
\left(U_{\alpha} y_{\gamma}\right)(t) \equiv x+\int_{0}^{t} d s F_{\alpha}\left(y_{\gamma}(s)\right) .
$$

Under the hypotheses $(\mathrm{p} 1)-(\mathrm{p} 4)$, and using the results of Section 3, we will now prove that $\left\{U_{\alpha}\right\}_{\alpha \in I}$ is an uniform family of $\operatorname{w} \tau \operatorname{sc}\left(\mathcal{L}_{\gamma}^{+}\right)$, which is also $\tau$-strong Cauchy.

First, it is evident that each $U_{\alpha}$ is a $\operatorname{w} \tau \operatorname{sc}\left(\mathcal{L}_{\gamma}^{+}\right)$.

Secondly, taking $y_{\gamma}, z_{\gamma} \in \mathcal{L}_{\gamma}^{+}$, we have $y_{\gamma}=e^{i H_{\gamma} t} y_{t} e^{-i H_{\gamma} t}$ and $z_{\gamma}=$ $e^{i H_{\gamma} t} z_{t} e^{-i H_{\gamma} t}$, where $y_{t}$ and $z_{t}$ belong to $\mathcal{L}^{+}(\mathcal{D})$ and could, in principle, depend on $t$. Therefore, using equations (4.24), (4.19) and the unitarity of the operators $\exp \left( \pm i H_{\gamma} s\right)$, we get

$$
\begin{aligned}
\|\left(U_{\alpha} y_{\gamma}\right)(t) & -\left(U_{\alpha} z_{\gamma}\right)(t) \|^{h, k} \\
& \leq \int_{0}^{t}\left\|\exp \left(i H_{\gamma} s\right)\left(F_{\alpha}\left(y_{s}\right)-F_{\alpha}\left(z_{s}\right)\right) \exp \left(-i H_{\gamma} s\right)\right\|^{h, k} \\
& =\int_{0}^{t}\left\|\left(F_{\alpha}\left(y_{s}\right)-F_{\alpha}\left(z_{s}\right)\right)\right\|^{h, k} d s .
\end{aligned}
$$


Since $\left\|\left(F_{\alpha}\left(y_{s}\right)-F_{\alpha}\left(z_{s}\right)\right)\right\|^{h, k}=\left\|h(H)\left[H_{\alpha}, y_{s}-z_{s}\right] H^{k}\right\|$, we have, inserting twice $H H^{-1}$ in each term below:

$$
\begin{aligned}
\|\left(F_{\alpha}\left(y_{s}\right)\right. & \left.-F_{\alpha}\left(z_{s}\right)\right) \|^{h, k} \\
& \leq\left\|h(H) H_{\alpha}\left(y_{s}-z_{s}\right) H^{k}\right\|+\left\|h(H)\left(y_{s}-z_{s}\right) H_{\alpha} H^{k}\right\| \\
& \leq 2\left\|H^{-1}\right\|\left\|H^{-1} H_{\alpha}\right\|\left\|y_{s}-z_{s}\right\|^{h_{+1}, k+1} \\
& \leq 2\left\|H^{-1}\right\|\left\|H^{-1} H_{\alpha}\right\|\left\|y_{\gamma}(s)-z_{\gamma}(s)\right\|^{h_{+1}, k+1} \\
& \leq 2\left\|H^{-1}\right\|\left\|H^{-1} H_{\alpha}\right\|\left\|y_{\gamma}-z_{\gamma}\right\|_{\delta}^{h_{+1}, k+1},
\end{aligned}
$$

where we have used again the unitarity of the operators $\exp \left( \pm i H_{\gamma} s\right)$ and the definition of \|\|$_{\delta}^{h, k}$. Finally, definition (4.15) gives

$$
\left\|U_{\alpha} y_{\gamma}-U_{\alpha} z_{\gamma}\right\|_{\delta}^{h, k} \leq c_{\alpha}\left\|y_{\gamma}-z_{\gamma}\right\|_{\delta}^{h_{+1}, k+1} .
$$

Of course, due to hypothesis (4.16), we also get that

$$
c_{-}=\lim _{\alpha \rightarrow 0} c_{\alpha}=\delta \lim _{\alpha \rightarrow 0} 2\left\|H^{-1}\right\|\left\|H^{-1} H_{\alpha}\right\|>0 .
$$

This is enough to conclude that $\left\{U_{\alpha}\right\}_{\alpha \in I}$ is an uniform family of $\mathrm{w} \tau \operatorname{sc}\left(\mathcal{L}_{\gamma}^{+}\right)$. To prove that it is also a $\tau$-strong Cauchy net, we have to check that $\| U_{\alpha} y_{\gamma}-$ $U_{\beta} y_{\gamma} \|_{\delta}^{h, k} \rightarrow 0$ for all $(h, k) \in \mathcal{C}_{N}$ and for any $y_{\gamma} \in \mathcal{L}_{\gamma}^{+}$when both $\alpha$ and $\beta$ go to zero.

Using the same procedure as above, we first obtain

$$
\left\|\left(U_{\alpha} y_{\gamma}\right)(t)-\left(U_{\beta} y_{\gamma}\right)(t)\right\|^{h, k} \leq \int_{0}^{t}\left\|F_{\alpha}\left(y_{s}\right)-F_{\beta}\left(y_{s}\right)\right\|^{h, k} d s .
$$

This implies, after some easy estimates, that

$$
\left\|U_{\alpha} y_{\gamma}-U_{\beta} y_{\gamma}\right\|_{\delta}^{h, k} \leq \delta\left\|\left[H_{\alpha}-H_{\beta}, y\right]\right\|_{\delta}^{h, k}
$$

and the rhs goes to zero because of the (4.17).

Therefore, we conclude that Proposition 3 can be applied. This means that the dynamics for the model can be obtained as a $\tau$-limit of the regularized dynamics, as obtained from the equation (4.25).

We end this section, and the paper, with an explicit $\mathrm{QM}_{\infty}$ model in which conditions (p1)-(p4) are satisfied. We refer to [9] for further details.

We take $\delta=1$. The starting point is the pair of the annihilation and creation operators $a, a^{\dagger}$, which satisfy the canonical commutation relation $\left[a, a^{\dagger}\right]=\mathbb{1}$. Let $N=a^{\dagger} a$ be the number operator (which we will identify 
with its self-adjoint extension), with spectral decomposition $N=\sum_{l=0}^{\infty} l E_{l}$. We take $N$ as the hamiltonian of the one mode free bosons. Of course, from the point of view of the dynamics, nothing change if we add a constant to the hamiltonian. Therefore we define, for reasons which will be clear in the following, $H=4 \mathbb{1}+N=\sum_{l=0}^{\infty}(4+l) E_{l}$. As in [9], we introduce an occupation number cutoff $H \rightarrow H_{L}=4 \mathbb{1}+Q_{L} N Q_{L}$, where $Q_{L}=\sum_{l=0}^{L} E_{l}$ is a projection operator. We can write $H_{L}=\sum_{l=0}^{\infty} c_{l}^{(L)} E_{l}$, where $c_{l}^{(L)}$ is equal to $4+l$ for $l=0,1,2, . ., L$ and is equal to 4 for $l>L$. We also have $H^{-1}=\sum_{l=0}^{\infty}(4+l)^{-1} E_{l}$ and $H^{-1} H_{L}=H_{L} H^{-1}=\sum_{l=0}^{\infty} b_{l}^{(L)} E_{l}$, where $b_{l}^{(L)}=c_{l}^{(L)} / 4+l$.

Obviously we have:

- $\left[H_{L}, H_{L^{\prime}}\right]=0, \quad \forall L, L^{\prime}$;

- since $\left\|H^{-1}\right\|=\frac{1}{4}\left(\leq \frac{1}{2(\delta+1)}=\frac{1}{4}\right)$ and $\left\|H^{-1} H_{L}\right\|=1$, as it can be easily checked, then

$$
\lim _{L \rightarrow \infty} 2\left\|H^{-1}\right\|\left\|H^{-1} H_{L}\right\|=\frac{1}{2}>0 ;
$$

- $\lim _{L \rightarrow \infty}\left\|\left[H-H_{L}, y\right]\right\|^{h, k}=0$. In fact we have

$$
\begin{aligned}
\|[H & \left.-H_{L}, y\right] \|^{h, k} \\
& \leq\left\|\left(H-H_{L}\right) y\right\|^{h, k}+\left\|y\left(H-H_{L}\right)\right\|^{h, k} \\
& \leq\left\|\sqrt{h(H)}\left(H-H_{L}\right)\right\|\left\|\sqrt{h(H)} y H^{k}\right\|+\left\|h(H) y H^{k+3}\right\|\left\|\left(H-H_{L}\right) H^{-3}\right\| .
\end{aligned}
$$

We know that, if $h \in \mathcal{C}$ then also $\sqrt{h} \in \mathcal{C}$. Since the function $h$ goes to zero faster than any inverse power, using the spectral decompositions for $H, H_{L}$ and $\sqrt{h(H)}$, it is easy to check that $\lim _{L \rightarrow \infty}\left\|\sqrt{h(H)}\left(H-H_{L}\right)\right\|=0$. Analogously it is not difficult to check that $\left\|\left(H-H_{L}\right) H^{-3}\right\|$ goes to 0 in the same limit.

In this way we have checked that for the free bosons all the points of the definition of a uniform family of $\mathrm{w} \tau \mathrm{sc} \tau$-strong Cauchy are satisfied, so that the existence of the thermodynamical limit of the model follows from the analysis proposed in this paper.

\section{$\S 5 . \quad$ Concluding Remarks}

In this paper we have discussed a possible extension of the notion of contraction map to a quasi *-algebraic framework, with particular reference to the existence of fixed points and to the continuity of contractions depending on a parameter. Both the mathematical and the physical interest of the subject is, in our opinion, quite evident. In particular, we believe that the possibility of setting up a new general approach for the problem of the existence of the dynamics for physical problems in many-body theory, quantum field theory or 
quantum statistical mechanics can be considered as a nice result, which deserves further studies. In particular, we believe that a deeper analysis of the set $\mathcal{B}_{L}$ is certainly worth. Also, a weakening of the hypotheses of Propositions 2 and 3 could be relevant in order to enlarge the class of models whose thermodynamical limit can be analyzed following the procedure proposed here. Finally, we plain to find additional conditions which ensure uniqueness of the fixed point and to consider the problem of the thermodynamical limit in the Schrödinger representation.

\section{Acknowledgments}

It is a pleasure to thank C. Trapani for his stimulating suggestions. This work has been supported by M.U.R.S.T.

\section{Appendix. On the Non-triviality of $\mathcal{B}_{L}$}

The proof of the existence of non zero elements in the set $\mathcal{B}_{L}$ is better carried out working with the topology $\tau_{0}$ we mentioned in Section 1.

The building block for defining this new topology is a subset $\mathcal{C}_{0}$ of $\mathcal{C}$. We start introducing a fixed positive real number, $m$, a finite subset of $\boldsymbol{N}, J$, and a corresponding set of positive real numbers, $\left\{x_{l}, l \in J\right\}$. Further, we define

$$
\mathcal{C}_{0} \equiv\left\{f \in \mathcal{C}: f\left(x_{l}\right) \leq m, l \in J\right\}
$$

It is evident from this definition that $\mathcal{C}_{0} \subset \mathcal{C}$. It is also clear that to any function $f(x) \in \mathcal{C}$ can be associated, in a non-unique way, a function $f_{0}(x) \in \mathcal{C}_{0}$ which is proportional to $f(x)$. It is enough to take this proportionality constant to be

$$
\tilde{k}=m \min _{l \in J^{\prime}}\left(f\left(x_{l}\right)^{-1}\right),
$$

where $J^{\prime}$ is the largest subset of $J$ such that $f\left(x_{l}\right) \neq O$ for all $l \in J^{\prime}$. (If $f\left(x_{l}\right)=O$ for all $l \in J$ then we can define $f_{0}(x)=0$.) We put $f_{0}(x)=\tilde{k} f(x)$.

$\tau_{0}$ is the topology defined by the following seminorms

$$
X \in \mathcal{L}^{+}(\mathcal{D}) \rightarrow\|X\|^{f, k} \equiv \max \left\{\left\|f(N) X N^{k}\right\|,\left\|N^{k} X f(N)\right\|\right\},
$$

where $k \geq 0$ and $f \in \mathcal{C}_{0}$. As for the topology $\tau$, we will consider only the first term above, $\left\|f(N) X N^{k}\right\|$. It is evident that the two topologies are indeed very close to each other. In fact, they are equivalent since the above construction implies that:

- all the seminorms of the topology $\tau_{0}$ are also seminorms of the topology $\tau$; 
- all the seminorms of the topology $\tau,\left\|f(N) X N^{k}\right\|$, can be written in terms of a seminorm of the topology $\tau_{0}, \tilde{k}\left\|f_{0}(N) X N^{k}\right\|$, where the functions $f$ and $f_{0}=\tilde{k} f$ belong respectively to $\mathcal{C}$ and $\mathcal{C}_{0}$.

For this reason $\mathcal{L}^{+}(\mathcal{D})$ turns out to be also $\tau_{0}$-complete. It is evident that the use of one or the other set of seminorms is completely equivalent and that this choice is only a matter of convenience. For instance, the use of $\tau_{0}$ simplifies the proof of the non-triviality of the set $\mathcal{B}_{L}$.

The first step of this proof consists in an analysis of the spectrum of the operator $H$ involved in the definition of the seminorms. We require to this unbounded self-adjoint operator to have a spectrum with a discrete part and with a finite number of eigenvalues $h_{i}$ with modulus not larger than 1 . We call $\mathcal{G}$ the set of the corresponding indices: $\left|h_{i}\right| \leq 1, \forall i \in \mathcal{G}$. For instance, if $N$ is the usual number operator, whose spectrum is $\{0,1,2,3,4, \ldots\}$, then the operator $H=(1 / 5) N$ satisfies the above condition with $\mathcal{G}=\{1,2,3,4,5,6\}$.

We use now the set $\left\{h_{i}\right\}_{i \in \mathcal{G}}$ and a positive real $m$ to define the set of functions $\mathcal{C}_{0}$ as above and, by means of $\mathcal{C}_{0}$, the topology $\tau_{0}$. The role of the hamiltonian in the construction of the topology here is evident, as in the original Lassner's paper, [6].

Calling $E_{l}$ the spectral projectors of the operator $H$, which from now on will be assumed to have discrete spectrum only to simplify the notation, we can write:

$$
H=\sum_{l=0}^{\infty} h_{l} E_{l}=\sum_{l \in \mathcal{G}} h_{l} E_{l}+\sum_{l \notin \mathcal{G}} h_{l} E_{l}
$$

which implies that

$$
H^{k}=\sum_{l \in \mathcal{G}} h_{l}^{k} E_{l}+\sum_{l \notin \mathcal{G}} h_{l}^{k} E_{l}, \quad h(H)=\sum_{l \in \mathcal{G}} h\left(h_{l}\right) E_{l}+\sum_{l \notin \mathcal{G}} h\left(h_{l}\right) E_{l} .
$$

Let now consider a set of complex number $\left\{c_{i}\right\}_{i \in \mathcal{G}}$ satisfying condition $\sum_{l \in \mathcal{G}}\left|c_{l}\right|$ $\leq \sqrt{L / m}, L>0$. Starting with this set we define the operator $Y \equiv \sum_{l \in \mathcal{G}} c_{l} E_{l}$. Our aim is to show that the operator $X \equiv Y^{2}=\sum_{l \in \mathcal{G}} c_{l}^{2} E_{l}$ belongs to some $\mathcal{B}_{L^{\prime}}$, at least under some conditions on the $\mathrm{w} \tau \mathrm{sc} T$.

First of all we consider the following inequality $\|X\|^{h, k} \leq\|h(H) Y\|\left\|Y H^{k}\right\|$. Secondly, we estimate separately the two contributions. We get

$$
\left\|Y H^{k}\right\|=\left\|\sum_{l \in \mathcal{G}} c_{l} E_{l}\left[\sum_{l \in \mathcal{G}} h_{l}^{k} E_{l}+\sum_{l \notin \mathcal{G}} h_{l}^{k} E_{l}\right]\right\| \leq \sum_{l \in \mathcal{G}}\left|c_{l}\left\|\left.h_{l}\right|^{k}\right\| E_{l} \| \leq \sum_{l \in \mathcal{G}}\right| c_{l} \mid,
$$


and, since $\left|h\left(h_{l}\right)\right| \leq m$ for all $l \in \mathcal{G}$,

$$
\begin{aligned}
\|h(H) Y\| & =\left\|\left[\sum_{l \in \mathcal{G}} h\left(h_{l}\right) E_{l}+\sum_{l \notin \mathcal{G}} h\left(h_{l}\right) E_{l}\right] \sum_{l \in \mathcal{G}} c_{l} E_{l}\right\| \\
& \leq \sum_{l \in \mathcal{G}}\left|c_{l}\right|\left|h\left(h_{l}\right)\right|\left\|E_{l}\right\| \leq m \sum_{l \in \mathcal{G}}\left|c_{l}\right| .
\end{aligned}
$$

Now, recalling the bound on the set $\left\{\left|c_{i}\right|\right\}_{i \in \mathcal{G}}$, we can conclude that

$$
\|X\|^{h, k} \leq m\left(\sum_{l \in \mathcal{G}}\left|c_{l}\right|\right)^{2} \leq L .
$$

Therefore, due to Lemma 1, point (a), we find that $X$ belongs to $\mathcal{B}_{L}$, at least if $T 0=0$. We get a similar conclusion even in the weaker hypothesis on $T$ of Lemma 1, point (b). Of course, it is not difficult to generalize this strategy in order to construct many other elements of $\mathcal{B}_{L}$.

\section{References}

[1] Smart, D. R., Fixed Point Theorems, Cambridge University Press, 1974.

[2] Reed, M. and Simon, B., Methods of Modern Mathematical Physics, I, Academic Press, New York, 1980.

[3] Kantorovich, L. V. and Akilov, G. P., Functional Analysis in Normed Spaces, Pergamon Press, 1964.

[4] Bagarello, F. and Morchio, G., Dynamics of Mean-Field Spin Models from Basic Results in Abstract Differential Equations, J. Stat. Phys., 66, 3/4 (1992), 849-866.

[5] Haag, R. and Kastler, D., An algebraic approach to Quantum Field Theory, J. Math. Phys., 5 (1964), 848-861.

[6] Lassner, G., Topological algebras and their applications in Quantum Statistics, Wiss. Z. KMU-Leipzig, Math.-Naturwiss. R., 30 (1981), 572-595; Algebras of unbounded operators and quantum dynamics, Phys. A, 124 (1984), 471-480.

[7] Antoine, J.-P. and Karwowski, W., Partial *-algebras of closed linear operators in Hilbert space, Publ. RIMS, Kyoto Univ., 21 (1985), 205-236.

[8] Bagarello, F. and Trapani, C., States and Representations of $C Q^{*}$-Algebras, Ann. Inst. H. Poinc., 61 (1994), 103-133.

[9] Bagarello, F., Applications of Topological *-Algebras of Unbounded Operators, J. Math. Phys., 66, 3/4 (1992), 849-866.

[10] - Applications of Topological *-Algebras of Unbounded Operators to Modified Quons, submitted to Publ. RIMS, Kyoto Univ.

[11] Bagarello, F. and Trapani, C., "Almost" Mean Field Ising Model: An Algebraic Approach, J. Stat. Phys., 65, 3/4 (1991), 469-482.

[12] - The Heisenberg Dynamics of Spin Systems: A Quasi*-Algebras Approach, J. Math. Phys., 37 (1996), 4219-4234. 